\title{
Montero Caro, María Dolores, Gobierno abierto como oportuni- dad de cambio, Dykinson, Madrid, 2020, 166 pp, ISBN 978-84- 1324-858-5.
}

\author{
http://dx.doi.org/10.18543/ed-68(2)-2020pp397-399
}

Hace escasos meses era noticia la aprobación por parte del pleno del Foro de gobierno abierto del IV plan de España sobre esta materia (20202024). En este aparecen marcados como principales objetivos la profundización en la transparencia y en la rendición de cuentas de las administraciones públicas, el impulso y consolidación de la participación en la gestión pública, el fortalecimiento de los valores éticos y de los mecanismos que afiancen la integridad de las instituciones públicas, y la sensibilización de la sociedad y empleados públicos sobre los valores del gobierno abierto. En un contexto de múltiples y diversas dificultades ocasionadas a raíz de la pandemia mundial que estamos atravesando, la colaboración entre las administraciones públicas, la ciudadanía, las organizaciones sociales y las entidades empresariales ha mostrado, en varias ocasiones, ser clave para abordar los desafíos culturales, políticos, económicos y jurídicos actuales.

Precisamente sobre el potencial y las limitaciones de la filosofía y herramientas del gobierno abierto versa el trabajo de la profesora María Dolores MONTERO CARO. En su monografía, la profesora de Derecho constitucional de la Universidad de Córdoba se sumerge en las posibilidades del gobierno abierto y, de manera especial, en las implicaciones de la transparencia para las democracias de nuestros días. Este trabajo se desarrolla a lo largo de siete capítulos en los que se presenta un recorrido que parte del sistema político derivado de la Constitución española de 1978 y llega hasta las oportunidades y los desafíos que el uso de la informática y del internet han introducido en nuestras democracias, sin olvidar las referencias a las crisis de desafección política que se han vivido en numerosos países en la última década ni las referencias internacionales a los países de nuestro entorno.

En el primer capítulo la autora analiza, entre otras, cómo la crisis económica de 2008 afectó a los fundamentos del estado del bienestar surgido a partir de la II guerra mundial y, especialmente, a las democracias occidentales. Esta realidad no iba a ser ajena a la situación española y la profesora identifica varias razones: un clima generalizado de falta de representatividad de la ciudadanía, inexistencia 
de una garantía plena y efectiva de los derechos sociales, un avance de los nacionalismos en determinadas autonomías y las diferencias notorias entre la sociedad actual y la que aprobó nuestra carta magna. No obstante, entiende que esta crisis de representatividad supone también un potencial transformador si va acompañado de políticas de regeneración democráticas que conviertan en realidades jurídicas lo que hasta ahora han sido, en buena medida, principios de buena fe de los poderes públicos.

En el siguiente capítulo aborda cómo la sociedad del conocimiento y de la información han dado lugar a un nuevo modelo de sociedad. Siguiendo a autores como BECK, GIDDENS, CASTELLS, DRUCKER O BELL, analiza el papel que tanto la información como el conocimiento han asumido en la actualidad y su articulación como elementos de transformación social, económica, política e institucional. No obstante, la autora identifica una serie de retos que se abren ante la configuración de un nuevo modelo social: el primero, la heterogeneidad de sociedades y la necesidad de abordar estos cambios sin desatender las particularidades de las mismas; el segundo, el surgimiento de una actividad delictiva en el plano informático que plantea desafíos para los estados muy significativos en términos de ciberseguridad y delitos informáticos; $y$ finalmente, el aumento de la participación de la ciudadanía, el debilitamiento de las estructuras sociales e institucionales que podríamos denominar clásicas y la pérdida de capacidad del estado de imponer su voluntad. Esto último se desprende del análisis de movilizaciones de los últimos años a lo largo del mundo como la «primavera árabe» el 15-M español, las numerosas protestas localizadas en América Latina, Cataluña o China.

El tercer capítulo profundiza sobre la noción del open government y los tres pilares definitorios del mismo, a saber: transparencia, participación y colaboración. La profesora Montero analiza cómo se están relacionado las TIC y las administraciones públicas, relaciones que están favoreciendo o que están alineadas con algunos de los fundamentos del gobierno abierto: mayor rapidez en la comunicación, mayor audiencia, posibilidad de intercambios directos con la ciudadanía, etc. (por ejemplo, con el uso de las redes sociales). Sin embargo, en España estas ventajas que ofrecen las TIC no se han trasladado aún al ámbito electoral con herramientas como el voto electrónico, lo que supondría, entre otras ventajas, una mayor facilidad en el recuento de los votos e incluso una reducción notable en los costes de los procesos electorales. Para ello, propone la autora una modificación de la LOREG que incluya las garantías suficientes de seguridad digital y reducción de la brecha digital, realidad esta que se observa en España y que supone uno de los principales inconvenientes para implementar medidas de gobierno abierto. Para finalizar este capítulo, destaca experiencias de open government en Estados Unidos y Reino Unido.

En el siguiente capítulo se describe «el papel de España como miembro de la alianza para el gobierno abierto», iniciativa surgida en 2011 y que ya aglutina a casi 80 países. Presta 
especial atención a los planes de acción de gobierno abierto presentados hasta el momento por España: 20122014, 2014-2016 y 2017-2019. Del primero al tercero observamos una mayor implicación por parte de nuestras autoridades pues si bien lo formulado en 2012 eran meras declaraciones de intenciones, en los siguientes se aprobó la Ley de transparencia, acceso a la información pública y buen gobierno y se constituyó el Foro de gobierno abierto, sucesivamente. Mención especial debe hacerse al IV plan de España en la materia cuya aprobación estaba prevista para el primer semestre de 2020 pero que, dada la situación de crisis sanitaria provocada por el COVID-19, en el momento en el que este trabajo vio la luz aún no había sido aprobado ${ }^{1}$.

En el quinto capítulo la profesora MONTERO aborda con especial profundidad uno de los pilares del objeto de estudio, la transparencia. Desde el origen de esta noción, se dibuja el mapa normativo internacional y europeo que regula el acceso a la información y la transparencia y se compara la legislación sobre libertad de información en Reino Unido y en Estados Unidos. Entiende la autora que la transparencia es un principio inspirador en el estado democrático -que deriva de la visibilidad de la actuación del poder público- pero que no puede hablarse de gobierno abierto como tal

${ }^{1}$ Puede consultarse el IV Plan de gobierno abierto de España (2020-2024) en el siguiente enlace: https://transparencia. gob.es/transparencia/dam/jcr:d306cd62cc0f-40a1-9be8-fe24eeee10d/IVPlanGobiernoAbierto-ES_2020-2024.pdf hasta el desarrollo de las nuevas tecnologías y de internet. Presenta a continuación el debate existente sobre la configuración de la transparencia bien como un derecho o bien como un principio. Insistiendo en que la transparencia es un principio fundamental de las democracias, la autora se identifica con quienes entienden que no puede ser configurado como un derecho de ejercicio individual ni de cuarta generación. Sobre la incorporación de este principio en el marco constitucional español, resultan relevantes los derechos de libertad de expresión, participación ciudadana, acceso a archivos y registros y los principios del ordenamiento jurídico. No obstante, la $\mathrm{CE}$ no recoge la transparencia como tal y esta se incorpora a nuestro ordenamiento jurídico a través de la legislación estatal y autonómica aprobada al respecto, normativa que, a pesar de ser relevante, según la autora presenta carencias como las presentes en la Ley 19/2013, de 9 de diciembre, de transparencia, acceso a la información pública y buen gobierno. Concretamente la profesora MONTERO denuncia la inexistencia de normativa acorde con la Directiva del Parlamento Europeo y el Consejo, de octubre de 2019, sobre protección de los denunciantes de corrupción.

En el siguiente capítulo se detiene sobre los otros dos pilares del gobierno abierto: la participación ciudadana y la colaboración. Sobre el primero, y tras presentar la teoría de ARSTEIN y la escalera de la participación, analiza los instrumentos de participación directa que se desprenden del texto constitucional español: participación directa por medio de representantes, 
referéndum, consultas populares autonómicas, referéndums locales y municipales, iniciativa legislativa popular e iniciativa ciudadana europea. Asimismo, se detiene sobre las formas de participación indirecta que se derivan también de la constitución: consejos de la juventud, organizaciones de consumidores y usuarios, participación en la empresa, etc. No obstante, entiende la autora que estos mecanismos resultan insuficientes en términos de participación y felicita las medidas de éxito adoptadas en el ámbito autonómico y local. Concretamente, se detiene en analizar tres: el portal Irekia del País Vasco por ser una iniciativa que tiene en cuenta los tres pilares del gobierno abierto, la iniciativa «Decide Madrid» que permite a los ciudadanos hacer propuestas de mejora de la ciudad y participar en la votación de asuntos públicos, y la Escuela de participación ciudadana de Córdoba por suponer un espacio relevante de formación e información de la ciudadanía. Sobre la colaboración, y siguiendo la teoría de CALDERÓN, se detiene en las plataformas crowdsourcing por permitir que la puesta en valor del conocimiento o las vivencias particulares a disposición de la vida pública.

Por último, la autora formula cinco planteamientos que considera que deben ser tomados en cuenta en el futuro inmediato. El primero, hace referencia al anhelo de participación de la ciudadanía en los asuntos que les afectan y una mayor exigencia de comunicación con los poderes públicos y que implica, también, la petición de responsabilidad de los cargos políticos cuando se extralimitan. Al respecto, insiste en la utilización de los mecanismos de participación popular a nivel municipal o local por darse en el plano de mayor cercanía entre instituciones y ciudadanía. En segundo lugar, propone la reforma de la $\mathrm{CE}$ para adaptarla a la configuración de internet como derecho humano, tal y como hizo la ONU en 2011. Aunque los estatutos de autonomía reformados sí han incorporado esta nueva realidad en su texto, nuestra carta magna sigue sin reflejar la realidad de las TIC e internet. Para ello, propone una nueva redacción del art. 20.1.2 CE, del 20.3 CE que entiendan este derecho y su relación con el desarrollo personal y social del individuo. Otra de las prioridades que señala la autora es la de la implementación real del principio de transparencia. Para ello, opta por una opción que define de prudente y que implica la incorporación de la transparencia en la CE como un principio rector de la política social y económica, principio informador de la legislación positiva, de la práctica judicial y de la actuación pública. Esta configuración se traduce en cambios necesarios en la redacción del art. 9.3 CE, en el art. 105 CE y en la Ley orgánica $6 / 2002$, de 27 de junio, de partidos políticos. En cuarto lugar, la profesora MONTERO insiste en la idea expuesta y defendida en su trabajo consistente en dejar de identificar gobierno abierto solo con transparencia. Los poderes públicos deben fomentar un cambio cultural en la ciudadanía para que sean comprendidas las ventajas y las posibilidades de esta forma de gobierno. Para caminar hacia ello, propone la autora la configuración, a nivel estatal, de una herramienta similar al IPC de Transparency 
International. Finalmente, no podía faltar la referencia al futuro incierto del gobierno abierto como consecuencia de la pandemia mundial. Actualmente las medidas relativas al objeto de estudio se encuentran paralizadas, pero entiende la autora que en los próximos meses podrán retomarse los compromisos pendientes.

Estamos, por tanto, ante un riguroso y ambicioso trabajo que aborda un tema de especial trascendencia y actualidad. Lo hace, además, desde una dimensión jurídica, pero incorporando aportes desde disciplinas como las ciencias políticas o la sociología que permiten al lector una mejor comprensión de la materia dentro de su complejidad. En estos momentos de incertidumbre en prácticamente todos los ámbitos de nuestra vida política y social, resulta inevitable traer los recuerdos de la experiencia de desafección política intensificada a raíz de la crisis de 2008 que no fue una crisis exclusivamente económica. Es palpable que, en esta ocasión, la crisis del COVID-19 tampoco se limita a la dimensión estrictamente sanitaria, sino que puede hacer tambalear -o lo está haciendo- los pilares que creíamos consolidados de nuestros modelos de convivencia. Este trabajo, a mi parecer, recoge claves de análisis y propuestas que, precisamente para el nuevo escenario político -local, nacional e internacionalque ya se ha comenzado a dibujar constituyen antídotos y remedios para los síntomas de enfermedad que vuelven a manifestar nuestros modelos democráticos.

Alicia Cárdenas Cordón

Profesora de Derecho constitucional Universidad de Córdoba 


\title{
Montero CARo, María Dolores, Gobierno abierto como oportuni- dad de cambio, Dykinson, Madrid, 2020, 166 pp, ISBN 978-84- 1324-858-5.
}

\author{
http://dx.doi.org/10.18543/ed-68(2)-2020pp397-399
}

\section{Copyright}

Estudios de Deusto es una revista de acceso abierto, lo que significa que es de libre acceso en su integridad. Se permite su lectura, la búsqueda, descarga, distribución y reutilización legal en cualquier tipo de soporte sólo para fines no comerciales, sin la previa autorización del editor o el autor, siempre que la obra original sea debidamente citada y cualquier cambio en el original esté claramente indicado

Estudios de Deusto is an Open Access journal which means that it is free for full access, reading, search, download, distribution, and lawful reuse in any medium only for non-commercial purposes, without prior permission from the Publisher or the author; provided the original work is properly cited and any changes to the original are clearly indicated. 